\title{
MPC Controller Design For Low Frequency Vehicle Longitudinal Vibration Suppression
}

\author{
Ting Zhang, Ying Huang ${ }^{*}$, Peilin Dai and Donghao Hao \\ School of Mechanical Engineering, Beijing Institute of Technology, 5 South Zhongguancun Street, Beijing, China
}

\begin{abstract}
The low frequency longitudinal vibration is one of the key factors that affect the drivability. Therefore, the analysis and control of the low frequency longitudinal vibration of the vehicle has always been a key issue in the vehicle Vibration and Harshness (NVH). In this paper, a new active control strategy for motor torque compensation is proposed to apply to the driveline's counterforce so as to realize the active suppression of low frequency vehicle longitudinal vibration. In the MPC controller, reasonable parameter selection can improve not only the system's closed-loop performance, but also its stability and robustness. Based on the design of vehicle low frequency longitudinal vibration controller, we choose and design some important control parameters (e.g., prediction time domain, control time domain and weighting factor) to assure the MPC controller achieve the best performance. Besides, we also study the impact of different control parameters by its results, finding out the general impact of the law. The simulation results show that the variable weights MPC controller can achieve better performance by selecting MPC controller parameters reasonably.
\end{abstract}

Keywords-low frequency longitudinal vibration; model predictive control; variable weights

\section{INTRODUCTION}

Low frequency vehicle longitudinal vibration, whose frequency is lower than $10 \mathrm{~Hz}$, is excited by driveline torsional vibration, mainly occurring in driving manoeuvres of rapidly acceleration or deceleration, starting, shifting and braking. Low frequency $(0-10 \mathrm{~Hz})$ longitudinal vibration is an important part of driveability[1]. Because the resonance frequency of the human organ is within this range, the passengers are extremely sensitive to the low frequency longitudinal vibration of the vehicle[2], making low frequency vehicle longitudinal vibration become an important reason for the passenger dissatisfaction.

There are two solutions to this phenomenon. One is to improve the mechanical components of the transmission system, e g, clutch, axle, etc., which will necessarily lead to additional vibration due to changes of the components. The other is to control the reference input torque of the motor, changing the actual output torque of the motor to reduce the vibration[3]. There has been many scholars using the second method to control the low frequency vehicle longitudinal vibration. $\operatorname{In}[4]$, a speed control strategy is proposed to reduce the vibration of the vehicle by controlling the vibration of the transmission system. Some literatures like [5], taking the actual wheel speed and the target wheel speed difference as the control target, use the LQ optimal control method to suppress the low frequency vehicle longitudinal vibration.
The methods mentioned above can solve the vibration problem of the transmission system in the Tip-in/Tip-out conditions. However, when the motor torque is compensated, the motor output torque cannot follow the driver's demand torque timely, which may reduce the acceleration performance. That we have to ensure the driving comfort and the acceleration performance at the same time makes the control become a multi-objective control problem. Because the Model Predictive Control (MPC) can handle multi-objective control with some optimality under the constraints[6], MPC is widely used in control problems.

Predictive control, using a heuristic optimization concept, gives designers the freedom to choose the form of optimal performance indicators, which is extremely different from traditional PID control and optimal control[7]. Therefore, for the same controlled object, the control results may completely different due to various performance indicators in control parameters. MPC control parameters are generally gotten by engineering experience and trial-and-error method because of the lack of standard or systematic method, which greatly increases the blindness of parameter design. In addition, the various selections of the performance parameters (such as the prediction time domain, control time domain, weighting matrix, etc.) will directly lead to different actual control effects, so the optimization of predictive control parameters is of great significance.

In this paper, a new active control strategy for motor torque compensation has been proposed to apply to a counterforce to the driveline to realize the active suppression of low frequency vehicle longitudinal vibration. We add vehicle acceleration performance to control target, which can improve vehicle driving comfort without sacrificing vehicle acceleration performance. The rest of the paper is organized as follows. In Section II, a control-oriented vehicle dynamics model is established in the MATLAB/Simulink environment. In Section III, the prediction equation is derived and a MPC controller is designed based on the model mentioned in the Section II. In Section IV, the selections of important control parameters in MPC are given. Furthermore, the general design rule of control parameters is drawn. Finally, the concluding remarks are given and the results are analyzed in Section V.

\section{SYSTEM MODELING}

The research object of this paper is e200, an electric vehicle of Beijing Hyundai. The whole construction of the transmission system is shown in Figure I The motor torque is successively transmitted to the transmission and the main reducer. At the 
differential gear, the transformed motor torque is passed to the drive shafts. Finally the wheels connect the driveline to the street.

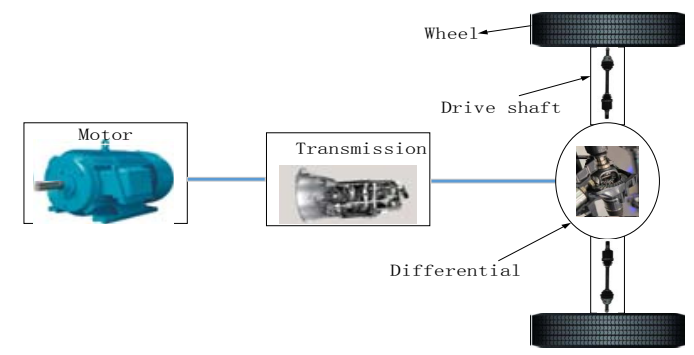

FIGURE I. THE CONSTRUCTION OF ELECTRIC VEHICLE TRANSMISSION SYSTEM

\section{A. Simplified Dynamics Model}

In order to design the model-based MPC controller to suppress the vibration of the vehicle, a simplified model for control should firstly be established so as to adjust control parameters conveniently. A simplified vehicle transmission system model is shown in Figure II

The whole drive train could be equivalent to two masses connected with a spring and damper. The moment of inertia from the motor to the differential gear is summarized to $J_{l}$ with viscous friction $d_{1}$ and the second moment of inertia $J_{2}$ comprises the remaining driveline and the vehicle with viscous friction $d_{2}$. The motor torque is represented by $T_{m}$, composed by request torque $T_{r}$ and compensated torque $T_{c}$. The product of the gearbox ratio $r_{g}$ and the differential gear ratio $r_{d}$ is denoted by $r$. It is assumed that the flexibility of drivetrain can be equivalent by a spring and a damper to be, represented by $k_{s}$ and $d_{s}$.

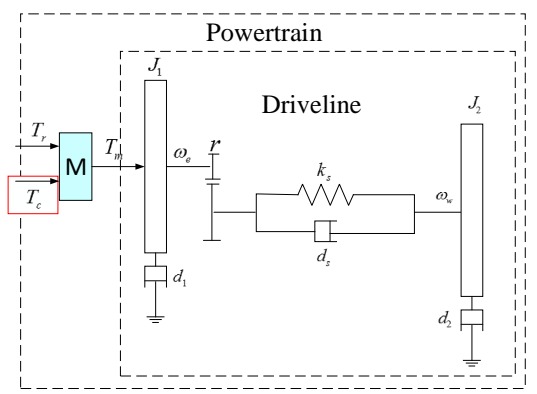

FIGURE II. SIMPLIFIED VEHICLE TRANSMISSION SYSTEM MODEL

The resistance of the vehicle mainly includes climbing resistance, wind resistance and tire rolling resistance. In this paper, the climbing resistance is ignored for that the road conditions in the simulation are straight roads. In the wind resistance calculation formula (1), $c_{x}, A, \rho$ and air velocity $v_{w}$ are relatively small, therefore wind resistance $F_{d}$ is too small to neglect at a low vehicle speed. The coefficients $f_{R 0}, f_{R 1}, f_{R 4}$ in the rolling resistance coefficient of the vehicle given by (2) are low to $10^{-2}$. At a low vehicle speed, the rolling resistance coefficient approximately equals $f_{R 0}$, which is generally less than 0.01 , hence it can also be ignored. The vehicle resistance is overlooked for the reason that vehicle has been in a lowspeed state in the simulation.

$$
F_{d}=c_{x} A \frac{\rho}{2}\left(v+v_{w}\right)^{2}
$$

$$
f_{R}=f_{R 0}+f_{R 1}\left(\frac{v}{100 k m / h}\right)+f_{R 4}\left(\frac{v}{100 k m / h}\right)^{4}
$$

The equivalent inertia of $J_{l}$ can be calculated by using (3) as below.

$$
J_{1}=J_{m}+J_{g 1}+J_{g 2} / r_{g}^{2}+J_{d} / r^{2}
$$

where, $J_{m}$ is inertia of motor

$J_{g l}$ is inertia of the input gear of the transmission

$J_{g 2}$ is inertia of the output gear of the transmission

$J_{d}$ is inertia of differential input gear

$r$ is belt transmission ratio

$r_{g}$ is gear transmission ratio below.

The equivalent inertia of $J_{2}$ can be calculated using (4) as

$$
J_{2}=J_{w}+m R^{2}
$$

Where, $J_{w}$ is inertia of wheel

$m$ is mass of vehicle body

$R$ is radius of tire

The equivalent stiffness of $k_{s}$ can be calculated using (5) as below. (The damping coefficient of $d_{s}$ is the same equation but replacing the stiffness coefficient by damping coefficient) The model acts as if there are several damped springs in series and parallel.

$$
\frac{1}{k_{s}}=\frac{1}{k_{p} r_{d}^{2}}+\frac{1}{k_{h 1}+k_{h 2}}
$$

Where, $k_{p}$ is stiffness coefficient of propeller shaft

$$
\begin{aligned}
& k_{h 1} \text { is stiffness coefficient of left half shaft } \\
& k_{h 2} \text { is stiffness coefficient of right half shaft }
\end{aligned}
$$

The dynamic of the drive train is shown in (6)-(8).

$$
\begin{gathered}
\mathrm{J}_{1} \dot{\omega}_{m}=T_{m}-d_{1} \omega_{m}-\frac{k_{s} \theta_{s}+d_{s} \dot{\theta}_{s}}{r} \\
\mathrm{~J}_{2} \dot{\omega}_{w}=-d_{2} \omega_{w}+k_{s} \theta_{s}+d_{s} \dot{\theta}_{s}
\end{gathered}
$$




$$
\dot{\theta}_{s}=\frac{\dot{\theta}_{m}}{r}-\dot{\theta}_{w}=\frac{\omega_{m}}{r}-\omega_{w}
$$

Where, $\theta_{s}$ is drive shaft torsion angle

$$
\begin{aligned}
& \omega_{m} \text { is motor speed } \\
& \omega_{w} \text { is wheel speed } \\
& \theta_{m} \text { is motor torsion angle } \\
& \theta_{w} \text { is wheel torsion angle }
\end{aligned}
$$

In order to apply control techniques, it is useful to write the equations in the state space. Drive shaft torsion angle $\theta_{s}$, motor speed $\omega_{m}$ and wheel speed $\omega_{w}$ are chosen as the state variables. The torsion speed, which is characterized as the vehicle jerk, is used as a virtual system output. The state space description of the dynamic system is like (9).

$$
\begin{gathered}
\dot{x}=A_{c} x+B_{c} T_{m} \\
\mathrm{y}=\mathrm{C}_{c} x
\end{gathered}
$$

$$
\begin{gathered}
\text { Where, } \mid x=\left[\begin{array}{lll}
\theta_{s} & \omega_{m} & \omega_{w}
\end{array}\right]^{T} \quad T_{m}=\left[\begin{array}{ll}
T_{r} & T_{c}
\end{array}\right]^{T} \\
\mathrm{y}=\dot{\theta}_{s}=\omega_{m} / r-\omega_{w}
\end{gathered}
$$

The parameters in (9) are shown as follow.

$$
\begin{gathered}
A_{c}=\left[\begin{array}{ccc}
0 & \frac{1}{r} & -1 \\
-\frac{k_{s} / r}{J_{1}} & -\frac{d_{1}+d_{s} / r^{2}}{J_{1}} & \frac{d_{s} / r}{J_{1}} \\
\frac{k_{s}}{J_{2}} & \frac{d_{s} / r}{J_{2}} & -\frac{d_{1}+d_{s}}{J_{2}}
\end{array}\right] \\
B_{c}=\left[\begin{array}{ccc}
0 & \frac{1}{J_{1}} & 0 \\
0 & \frac{1}{J_{1}} & 0
\end{array}\right]^{T} \quad C_{c}=\left[\begin{array}{lll}
0 & \frac{1}{r} & -1
\end{array}\right]
\end{gathered}
$$

\section{B. Discrete System Model}

Using the forward Euler method to transform the continuous system to discrete system, Laplace variable $\mathrm{s}$ is replaced using (10)

$$
s=\frac{z-1}{t_{s}}
$$

Using (10), the system model (9) can convert to discrete system. Equation (11) is the discrete state equation of (8). The coefficient is shown in (12).

$$
\begin{gathered}
x(k+1)=A x(k)+B T_{m}(k) \\
y(k)=C x(k) \\
A=I+A_{c} t_{s} \quad B=B_{c} t_{s} \quad C=C_{c}
\end{gathered}
$$

\section{Model Verification}

There exists a simulation credibility problem whether the control-based model really can reacts full dynamic process of object model. In order to minimize the influence of the modeling error, model verification is necessary. We check the control model based on the object model. The object model takes into account the motor inertia, gearbox ratio, main reducer ratio, gear inertia, gear drive efficiency, stiffness and damping of drive shaft and drive axle, tire inertia, tire stiffness and damping, adhesion coefficient, slip ratio, wind resistance and rolling resistance.

We give the object model and the control-oriented model the same initial simulation conditions. The vehicle is in first gear, quickly stepping on the accelerator pedal and then the motor torque will increase rapidly. The simulation results are shown in Figure IV.

Figure. IV show that the torsional speed of the control model can well track that of the object model and reflect the vibration of the object model to a certain extent. Within the range of error permitting, the control model can commendably reflect the dynamic performance of the object model and hence can be used in the control.

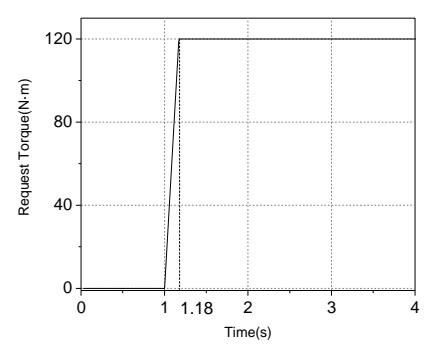

FIGURE III. REQEST TORQUE OF MOTOR

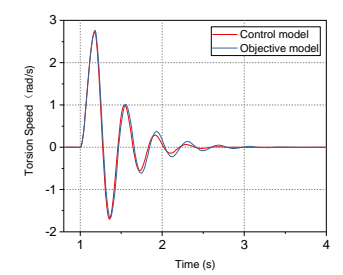

FIGURE IV. TORSION SPEEDOF TWO SYSTEMS

The Simulated vehicle is a motor vehicle. The parameters used in controller are shown in Table I. 
TABLE I. VEHICLE PARAMETERS

\begin{tabular}{|c|c|c|}
\hline Symbol & Parameters & Value \& Unit \\
\hline $\boldsymbol{J}_{\boldsymbol{1}}$ & $\begin{array}{c}\text { moment of inertia from the motor to the } \\
\text { differential gear }\end{array}$ & $0.10 \mathrm{~kg} \cdot \mathrm{m} 2$ \\
\hline $\boldsymbol{J}_{\boldsymbol{2}}$ & $\begin{array}{c}\text { moment of inertia of remaining } \\
\text { driveline and the vehicle }\end{array}$ & $67.328 \mathrm{~kg} \cdot \mathrm{m} 2$ \\
\hline $\boldsymbol{d}_{\boldsymbol{1}}$ & $\begin{array}{c}\text { damping of transmission and final } \\
\text { drive to bearings }\end{array}$ & $0.017 \mathrm{Nm} / \mathrm{rad} / \mathrm{s}$ \\
\hline $\boldsymbol{d}_{\boldsymbol{2}}$ & damping of wheels to bearings & $51.38 \mathrm{Nm} / \mathrm{rad} / \mathrm{s}$ \\
\hline $\boldsymbol{k}_{\boldsymbol{s}}$ & damping of the drive shaft & $4223.30 \mathrm{Nm} / \mathrm{rad}$ \\
\hline $\boldsymbol{d}_{\boldsymbol{s}}$ & Stiffness of the drive shaft & $78.90 \mathrm{Nm} / \mathrm{rad} / \mathrm{s}$ \\
\hline $\boldsymbol{r}$ & transmission ratio of drive train & 12 \\
\hline
\end{tabular}

\section{CONTROLLER Design}

Since MPC scheme needs full state variables for prediction in look-ahead horizon, Kalman filter is applied to the measurements to estimate the unmeasurable state variables. Fig. $\mathrm{V}$ shows an overall control structure using MPC approach with Kalman filter.

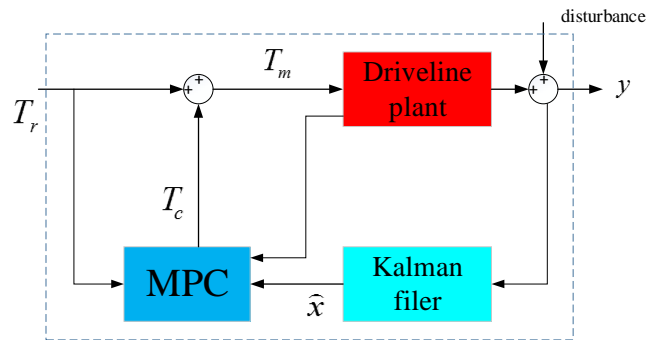

FIGURE V. SCHEMA OF OVERALL CONTROL SYSTEM

\section{A. Kalman Filter}

According to the method given in literature [8], the Kalman filter is designed to estimate the torsion angle based on the measured motor speed and wheel speed. The estimated formula is shown in (13).

$$
\hat{\dot{x}}_{k}=A_{k} \widehat{x}_{k}+B_{k} T_{m}+K_{k}\left(y-C_{k} \widehat{x}_{k}\right)
$$

The $K_{k}$ is a Kalman filter coefficient and is calculated using the algebraic Raccati Equation to minimize the estimation error. The process is overlooked in this paper.

\section{B. Control Target Setting}

Because the low frequency vehicle longitudinal vibration seriously affects the driving comfort, the purpose of this paper is to use the MPC controller to suppress the vibration of the transmission system. The torsional speed, which is characterized the vehicle jerk, is chosen as the control objective and regulated to zero to suppress the vibration. MPC controller is used to calculate the motor compensation torque, which is bound to affect the acceleration performance of the vehicle. So this article considers not only driving comfort but also acceleration performance, specifically described as follows.

- Driving comfort improvement, which is similar to minimize the torsion speed.

- Acceleration performance means vehicle response the driver's demand exactly. In this aspect, the controller output should not have a significant effect on the driver's demand torque, which is similar to minimize the output of the controller $T_{c}$.

The control objectives are written in (14)

$$
\min _{T_{c}(k)} J=\left\|W_{y} y\right\|^{2}+\left\|W_{u} T_{c}\right\|^{2}
$$

$W_{y}$ and $W_{u}$ are weighting factors of driving comfort and acceleration performance.

\section{Model Predictive Control}

According to the main principle of model prediction [9], using the state vector $x(k)$ as the initial condition of time $k$, the future torsional speed of the system is predicted based on the model (12) In this paper, the prediction step is represented by $P$, and $M$ is the control step, satisfying $M \leqslant P$. In order to derive the prediction equation of the system, the assumptions are set as below.

- Beyond control horizon, the control variables kept unchanged.

- Demand Torques $T r$ kept unchanged in prediction horizon

We define the vector $Y(k+1 \mid k)$ in (15), and the vector $U(k)$ in (16), which is the predicted input vector in horizon $M$.

$$
Y(k+1 \mid k) \square\left[\begin{array}{c}
\mathrm{y}(k+1 \mid k) \\
\vdots \\
\mathrm{y}(k+p \mid k)
\end{array}\right]
$$

$$
U(k)=\left[\begin{array}{lll}
T_{c}(k) & \cdots & T_{c}(k+m-1)
\end{array}\right]^{T}
$$

The cost function (14) can be defined as (17)

$$
\begin{aligned}
& \min _{U(k)} J(U(k), Y(k+1 \mid k)) \\
& =\left\|W_{y} Y(k+1 \mid k)\right\|^{2}+\left\|W_{u} U(k)\right\|^{2} \\
& =\sum_{i=1}^{P}\left\|W_{y}(\mathrm{y}(k+i \mid k)-r(k+i))\right\|^{2}+\sum_{i=1}^{M}\left\|W_{u} T_{c}(k+i-1)\right\|^{2}
\end{aligned}
$$

Solving the cost function (19) can convert to a quadratic programming problem (QP). Based on the method provided in Ref. [9], we can use the prediction equation (16) to obtain the solution of (19). 


\section{Selection ANd Design of Model Predictive CONTROL PARAMETERS}

After the MPC controller is built, the controller parameters are selected and adjusted. Whether the control strategy control effect is good or bad directly relates to parameter adjustment.

\section{A. Selections of the Prediction Horizon}

The length of the prediction horizon directly affects the computational efficiency of the whole control algorithm. As the prediction horizon increases, the robust stability of the system becomes better, but the corresponding computational efficiency will become low. When the prediction horizon is too short, although the computational efficiency is high, the control result may be poor due to the lack of future information. Giving the test object Tip-in process as the simulation condition, the control horizon $\mathrm{M}$ is fixed 6 and the prediction horizon is given in different values. Motor demand torque is shown in Figure III, and the control effects of the system under different prediction horizon is shown in Figure VI and Table II.
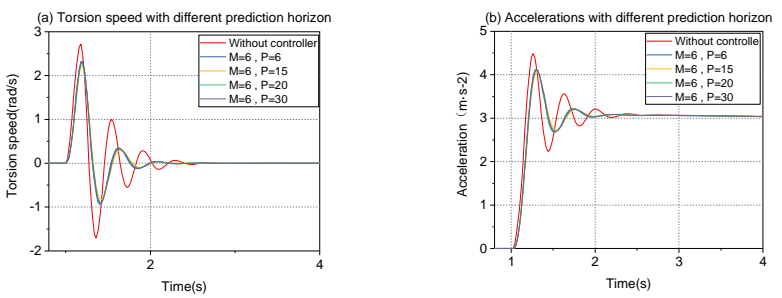

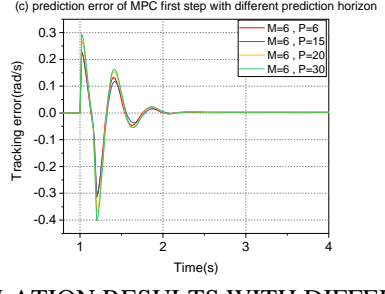

FIGURE VI. SIMULATION RESULTS WITH DIFFERENT PREDICTIO HORIZON

Seeing from Fig. VI and Table II, the response time increases with the increasing of the prediction horizon, and the control effect of the system is different under variable prediction horizon. It can be obtained from Table II, when prediction horizon is chosen 15 , the corresponding torsion speed fluctuations and acceleration fluctuations have a minimum value, that is to say, the vehicle vibration is controlled better and the driving comfort is relatively higher on a certain extent. There are the reasons.

1) When the the prediction horizon is small, the system obtains too little future information to make action properly. As the prediction horizon increases, the system can get more future operating state information, and the system can more reasonably calculate the compensation torque based on the predicted value.

TABLE II SYSTEM PERFORMANCE WITH DIFFERENT PREDICT HORIZON

\begin{tabular}{|c|c|c|c|}
\hline \multirow{2}{*}{$\begin{array}{c}\text { Prediction } \\
\text { horizon }\end{array}$} & \multicolumn{3}{|c|}{ Torsion speed } \\
\hline & Maximum & Minimum & Range \\
\hline 6 & 2.324 & -0.942 & 3.266 \\
\hline 15 & 2.221 & -0.831 & 3.052 \\
\hline 20 & 2.273 & -0.886 & 3.159 \\
\hline 30 & 2.295 & -0.911 & 3.206 \\
\hline \multicolumn{4}{|c|}{ Acceleration } \\
\hline 6 & 4.138 & 2.671 & 1.467 \\
\hline 15 & 4.072 & 2.727 & 1.345 \\
\hline 20 & 4.115 & 2.697 & 1.418 \\
\hline 30 & 4.130 & 2.682 & 1.448 \\
\hline
\end{tabular}

TABLE III SYSTEM PERFORMANCE WITH DIFFERENT CONTROL HORIZON

\begin{tabular}{|c|c|c|c|}
\hline \multirow{2}{*}{$\begin{array}{l}\text { Control } \\
\text { horizon }\end{array}$} & \multicolumn{3}{|c|}{ Torsion speed } \\
\hline & Maximum & Minimum & Range \\
\hline 2 & 1.390 & -0.169 & 1.559 \\
\hline 3 & 1.238 & -0.081 & 1.319 \\
\hline 6 & 2.092 & -0.771 & 2.863 \\
\hline 12 & 2.156 & -0.845 & 3.001 \\
\hline \multicolumn{4}{|c|}{ Acceleration } \\
\hline 2 & 3.438 & 3.060 & 0.378 \\
\hline 3 & 3.289 & 3.090 & 0.199 \\
\hline 6 & 4.015 & 2.751 & 1.264 \\
\hline 12 & 4.044 & 2.717 & 1.327 \\
\hline
\end{tabular}

2) When we derive the prediction equation, we have the assumptions that demand torques $T_{r}$ kept unchanged in prediction horizon. In the Tip-in process, the demand torque is always rising, so assumptions are false with the increase of the prediction horizon. As a result, the forecast will become inaccurate and the control effects are worse.

3) The rapidity of numerical solution optimization problems has been a key issue in predictive control applications. As the prediction horizon increases, the computational time in the calculation process increases, and the response time increases, resulting in poor control results.

In the controller design of this paper, we select the prediction horizon $P=15$. 


\section{B. Selections of the Control Horizon}

The control horizon is also an adjustable parameter, and more degrees of freedom are provided to the controller optimization with the addition of $M$. The smaller the $\mathrm{M}$ is, the worse the tracking performance of the system at each sampling point becomes. Select the fixed prediction horizon $P=15$ and give a different control horizon. Given the same simulation conditions as described in Section 4.1, the control effects of the system under different control horizon is shown in Figure VI and Table III.

It can be seen from Fig. VII and Table III that the control effect is exceedingly different with various values of the control horizon, mainly acting in the torsion speed fluctuation and acceleration fluctuation. From Fig. VI (a), when P is 3, the speed difference is the smallest, then the fluctuation becomes larger and the control effect is worse with the change of the control horizon. From Fig. 4-2b, the acceleration fluctuation is the smallest when $\mathrm{P}$ is 3 , and the fluctuation becomes larger as the control horizon changes, beside the response time becomes shorter and the response becomes better with the increase of control horizon. There are the reasons.

1) In the case where the prediction horizon $P$ is determined, the smaller the control horizon $M$ is, the worse the tracking performance of the system at each sampling point. Physically speaking, in order to achieve the desired output of the future P-times, it is necessary to share the P-expected tasks into $M$ optimization variables. When $M$ is particularly small, this goal is impossible for complex objects. So it is not difficult to understand, when $M$ is small, the control of the mobility is poor, that is to say, the dynamic performance of the system may not be satisfied, but the system's robust stability is often better.
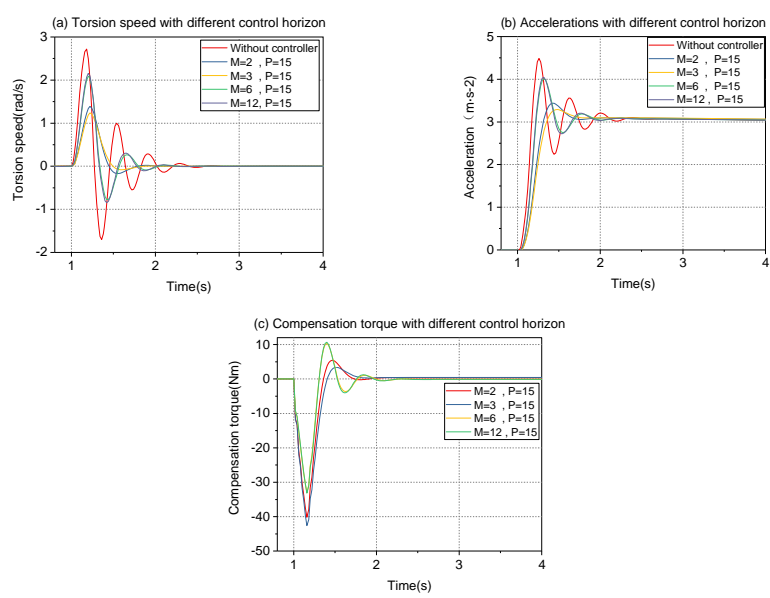

FIGURE VII. SIMULATION RESULTS WITH DIFFERENT PREDICTION HORIZON

2) When $M$ is large, the control system is of great mobility, and the overall dynamic performance of the system is improved, but it is easy to produce robust stability problem.

3) According to the optimization function (19) we can see that when $P$ is certain and $M$ is too large, the number of the independent variable will become more. To minimize the cost function $J$, the absolute value of the $T_{c}$ calculated by the MPC becomes smaller, which means that the compensation torque becomes smaller. For the process of torque rise, the compensation value is small and the slope of the motor's actual output torque is relatively large. As a reset, the driving comfort is relatively poor and the acceleration is relatively good.

In the controller design of this paper, we select the control horizon $M=3$.

\section{Modification of Multi - objective Optimization Weights}

The role of the weights of the controlled variable in the predictive control is that the user can select the weights to determine the importance of the controlled variable. The weights $W u$ and $W y$ are relative, and the effect of increasing one of the weighting matrices is equivalent to reducing the other weighting matrix. The two weighted matrices simultaneously increase the same multiple or shrink the same multiple has no effect on the optimal control law of the system. In order to conveniently design controller, we define $W u$ a diagonal matrix whose diagonal value is 1 . We give $W y$ three sets of parameters, two fixed values and one is changing, giving it a small value in the torque rise stage and then making it gradually rise to a relatively large value at the end of the vehicle torque rising. Given the same simulation conditions as described in Section 3.1 and $P$ is $15 M$ is 3, the simulation results are shown in Figure VII.

From Fig. VII and Table IV, with the increase of the weighting factor $W_{y}$, the fluctuation range of the torsion speed and acceleration in the time domain becomes smaller, which means the driving comfort of the system becomes better. At the same time, the time requiring for the peak time in the acceleration is also increased and the acceleration performance of the system is deteriorated. There are the reasons.
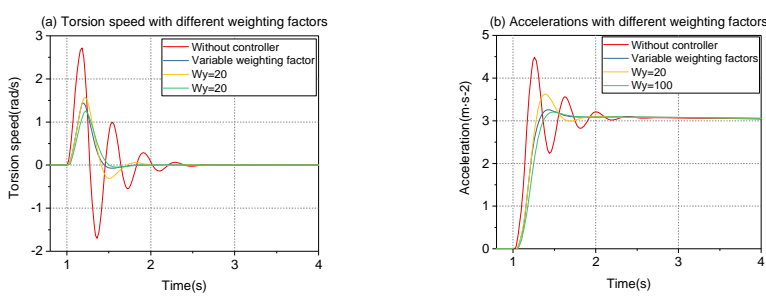

FIGURE VIII. SIMULATION RESULTS WITH VARIABLE WEIGHTING FACTORS

Increase the value of $W y$ and keep $W u$ unchanged is equivalent to enhance the importance of driving comfort. The resulting effect is that the torsion speed fluctuations and the acceleration fluctuations of the system become smaller, suppressing system vibration and improving the driving comfort to some extent. At the same time because the value of $W u$ is relatively small, changes of controlled quantities or energy consumption is open, and control costs rise. The compensating torque in the Tip-in process is negative, and the great absolute value of the compensating torque means sacrificing a certain degree of vehicle dynamics.

It can be seen from Fig.4-3, when we give the system variable weighting factor, both the acceleration performance and the driving comfort are guaranteed. 


\section{CONCLUSIONS}

Through the mechanism analysis, this paper first establishes the control model, and the model is checked to ensure the correctness of the model. Then the MPC controller is designed for solving the problem of the low frequency vehicle longitudinal vibration. Finally, through the design of the control parameters in the MPC, the control effect is achieved in a relatively good state.

1) When $P$ is given a small value, the system is less effective in controlling for the sake of future information, and the control effect is deteriorated due to the inaccuracy prediction with a large value of $\mathrm{P}$. So when we choose the parameter of the prediction horizon, it must be greater than time-delayed and time-tagged, and it is best to cover the main dynamic part of the object. And then combining the actual production requirements, the selection of $\mathrm{P}$ needs appropriate regulation, if the dynamic is not enough, the $\mathrm{P}$ should be appropriately reduced, if the stability is poor, the $\mathrm{P}$ should be appropriately increased.

2) System results are largely various because of different control horizon. When $M$ is given a small value, the tracking performance of the system at the sampling point is relatively poor and the response time is longer. When $M$ is given a large value, the dynamic response of the system becomes better, but the system stability is worse. So when we choose the parameter of the control horizon $M$, it must be less than or equal to prediction horizon $\mathrm{P}$, and as small as possible with satisfying the dynamic response.

3) The weighting factors $W u$ and $W y$, which are mutually constrained, correspond to the importance of the controlled variable. When we pay more attention to the acceleration performance, we can reduce the $W y$ (or increase $W u$ ) to achieve the control target. In order to reduce control costs and enhance the driving comfort, you can increase $W y$ (or reduce $W u$ ) to achieve. In order to trade off the acceleration performance and driving comfort in different control stage, the variable weighting factor strategy was proposed and verified. We give different values of $W y$ (or $W u$ ) at different control stage to correspond to the control problem, so that the MPC controller can better meet our expectations. Simulation results show that both performance are guaranteed.

\section{ACKNOWLEDGMENTS}

This research is supported by the National Science Foundation of China (grant No.51475043).

\section{REFERENCES}

[1] J. Li, M.G. Ouyang, A Study on Surge Control Strategy for Diesel Powertrain, Automotive Engineering, Vol.28, No.3, 238-241, 2006.

[2] Griffin, M. J. Handbook of human vibration. Handbook of hu man vibration /. Academic Press, 1990:2213.

[3] Zulkifli, S. A., N. Saad, and A. R. A. Aziz. "Deterministic and fuzzy logic control of through-the-road split-parallel hybrid electric vehicle." International Conference on Computer, Control, Informatics and ITS Applications IEEE, 2015:169-174.

[4] Pettersson, Magnus, and L. Nielsen. "Diesel-Engine Speed Control with Handling of Driveline Resonances." Control Engineering Practice, Vol.11, No.3, 319-328, 2003.
[5] X. Lu, Design of model predictive controller for anti-jerk during tipin/out process of vehicles, 30th Chinese Control Conference, 3395-3400, 2011.

[6] Saerens, Bart, et al. "Model predictive control of automotive powertrains - first experimental results." Decision and Control, 2008. Cdc 2008. IEEE Conference on IEEE, 2008:5692-5697.

[7] Rawlings, J B, and D. Q. Mayne. "Model Predictive Control: Theory and Design." 2009:3430-3433.

[8] Qi W, Zhang P, Deng Z. Robust weighted fusion Kalman filters for multisensor time-varying systems with uncertain noise variances [J] Signal Processing, 2014, 99: 185-200.

[9] H. Chen, Model predictive control. Beijing: Science Press, 2013.Pri 